\title{
Silicon Taper Based D-band Chip to Waveguide Interconnect for Millimeter-wave Systems
}

\author{
Ahmed Hassona, Vessen Vassilev, Zhongxia Simon He, Chiara Mariotti, Franz Dielacher and Herbert \\ Zirath
}

\begin{abstract}
This paper presents a novel interconnect for coupling Millimeter-wave $(\mathrm{mmW})$ signals from integrated circuits to air-filled waveguides. The proposed solution is realized through a slot antenna implemented in embedded Wafer Level Ball Grid Array (eWLB) process. The antenna radiates into a high-resistivity (HR) silicon taper perpendicular to its plane, which in turn radiates into an air-filled waveguide. The interconnect achieves a measured average insertion loss of $3.4 \mathrm{~dB}$ over the frequency range 116-151 $\mathrm{GHz}$. The proposed interconnect is generic and does not require any galvanic contacts. The utilized eWLB packaging process is suitable for low-cost high-volume production and allows heterogeneous integration with other technologies. This work proposes a straightforward cost-effective high-performance interconnect for mmW integration and thus addressing one of the main challenges facing systems operating beyond $100 \mathrm{GHz}$.
\end{abstract}

Index Terms-D-band, interconnects, waveguide, transition, taper, eWLB, millimeter waves, THz.

\section{INTRODUCTION}

$\mathrm{T}$ he increasing need for higher data rates makes $\mathrm{mmW}$ technologies an attractive solution for wireless applications and imaging systems [1].

In this regard, one of the main challenges facing full realization of such systems is the lack of low-cost interconnects to achieve high integration without compromising performance. Many techniques are proposed in literature to couple the RF signal to MMIC at $\mathrm{mmW}$ range. One possibility is to couple the MMIC directly to a waveguide and hence achieve high coupling over wide frequency band [2]. The drawback of this technique is that, most highly integrated circuits are relatively large in size and waveguide dimensions shrink as frequency goes higher and hence the MMIC width would exceed the subcritical dimension needed to prevent waveguide modes from propagating through the substrate making the integration of MMIC-to-waveguide transitions on-chip impractical. To mitigate this problem, Deal et al. suggested using a non-rectangular MMIC [3]. The presented solution requires complicated post-processing and

This project has received funding from the European Union's Horizon 2020 research and innovation program under grant agreement No. 644039.

Ahmed Hassona, Vessen Vassilev, Zhongxia Simon He and Herbert Zirath are with Microwave Electronics Lab., Chalmers University of Technology, Gothenburg, Sweden (e-mail: hassona@chalmers.se; vessen.vassilev@chalmers.se; zhongxia@chalmers.se; herbert.zirath@chalmers.se). Chiara Mariotti and Franz Dielacher are with Infineon Technologies Austria AG, Villach, Austria (e-mail: chiara.mariotti@infineon.com; franz.dielacher@infineon.com) wastes silicon area that can be utilized. Another approach is to use a separate transition [4] [5]. Using a separate transition is crucial from performance perspective, as coupling the RF signal directly to the MMIC from the waveguide causes waveguide modes to leak into the circuit cavity and hence affect performance. The drawback of this solution is that it requires bondwire connections between the waveguidetransition and the MMIC. Using bondwires at frequencies beyond $100 \mathrm{GHz}$ requires special compensation techniques [6] because of the high inductive behavior of bondwires at these frequencies.

This work addresses mmW system integration challenges by utilizing eWLB packaging technology. eWLB packaging provides an attractive solution for integrating MMIC into a mountable module with flexible I/O connections. eWLB processes have been used for packaging integrated circuits operating at frequencies up to $100 \mathrm{GHz}$ [7] [8]. However, pushing the technology beyond $100 \mathrm{GHz}$ has not been attempted before. In this work, Infineon's eWLB technology is used to implement a D-band slot antenna to couple the RF signal to a waveguide and at the same time providing both RF and DC connectivity to the MMIC through its redistribution layers and vias. The proposed approach is generic and can be used for integration with any MMIC and the concept is supported and verified by experimental results.

Figure 1 shows the proposed complete system which

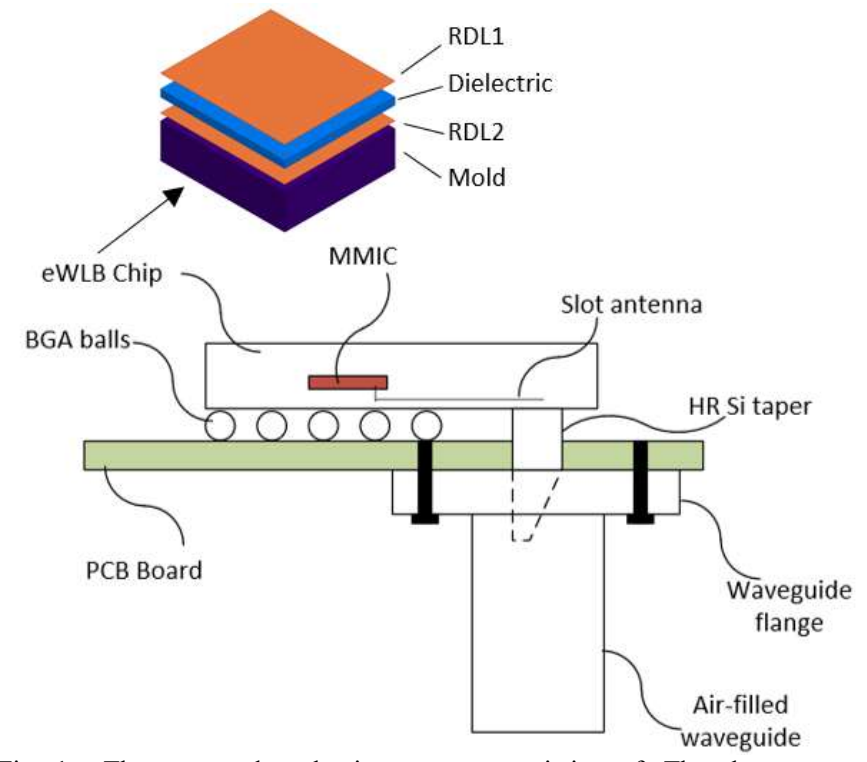

Fig. 1. The proposed packaging system consisting of: The slot antenna implemented on an eWLB chip, MMIC, PCB, HR Si taper and an air-filled waveguide. 
consists of an eWLB chip, MMIC, PCB, HR silicon taper and an air-filled WR-6.5 waveguide. The eWLB chip provides two metal layers named redistribution layers (RDL) that lie on the surface of a plastic mold. In this work, the RDLs are not only used to provide DC and low frequency connectivity to the MMIC through BGAs but also to implement a slot antenna which radiates the RF signal to a HR silicon taper perpendicular to its plane which is then used to guide the signal to an air-filled standard size D-band waveguide as shown in Figure 1. This work focuses on the high frequency portion of the system as will be discussed in detail in the following section.

The choice of eWLB technology is motivated by the need of a cost-effective and high-volume process for interconnects that operate beyond $100 \mathrm{GHz}$. This has been a major challenge hindering full implementation of $\mathrm{mmW}$ systems.

\section{INTERCONNECT DESIGN}

Figure 2 shows the implemented configuration. A D-band slot antenna is fabricated on Infineon's eWLB process. The antenna is implemented on RDL1 and fed through coplanar waveguide $(\mathrm{CPW})$ lines. The slot dimensions are chosen to provide reasonable impedance matching to the silicon taper and minimize radiation leakage at the interface. The taper is machined using a micro-dicing saw and its dimensions are shown in Figure 2. The taper is mounted normal to the slot and is then inserted into the air-filled waveguide as shown in Figure 2. The use of the Si taper helps guide the field to the air-filled waveguide and reduces leakage to the substrate due to the high dielectric constant of $\mathrm{Si}$. In order to provide mechanical support for the taper, a steel metal holder was machined to ensure accurate positioning with respect to the air-filled waveguide. The taper slides through a hole in the holder that has the same dimensions of the taper. The taper is then glued to the holder using Glycol Phthalate Wax and the holder is attached to the air-filled waveguide using conductive

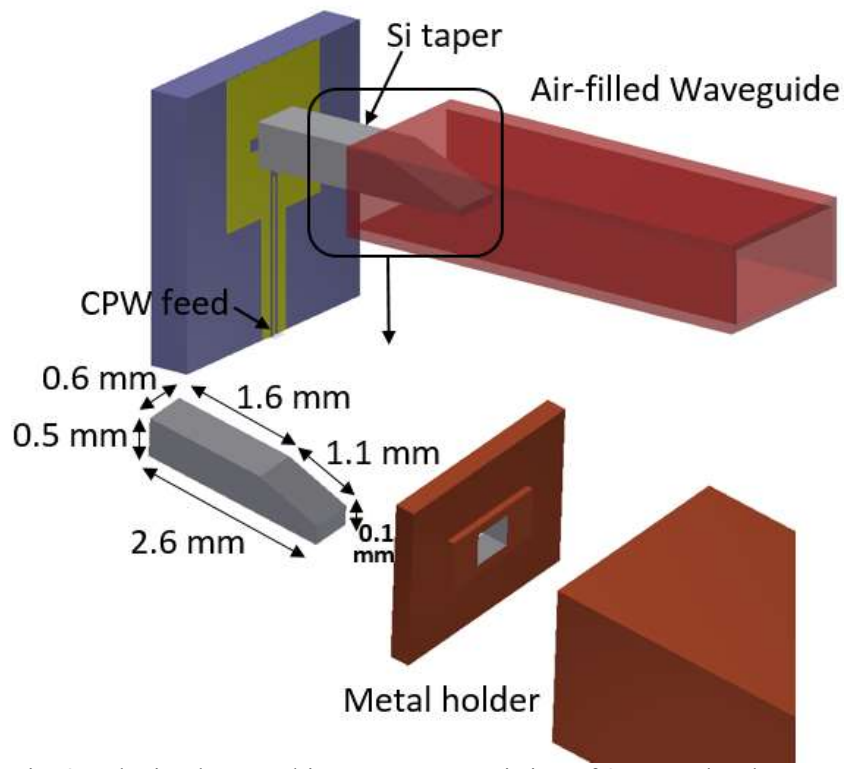

Fig. 2. The implemented interconnect consisting of 3 parts: the slot antenna implemented on eWLB chip, HR Si taper and air-filled waveguide.

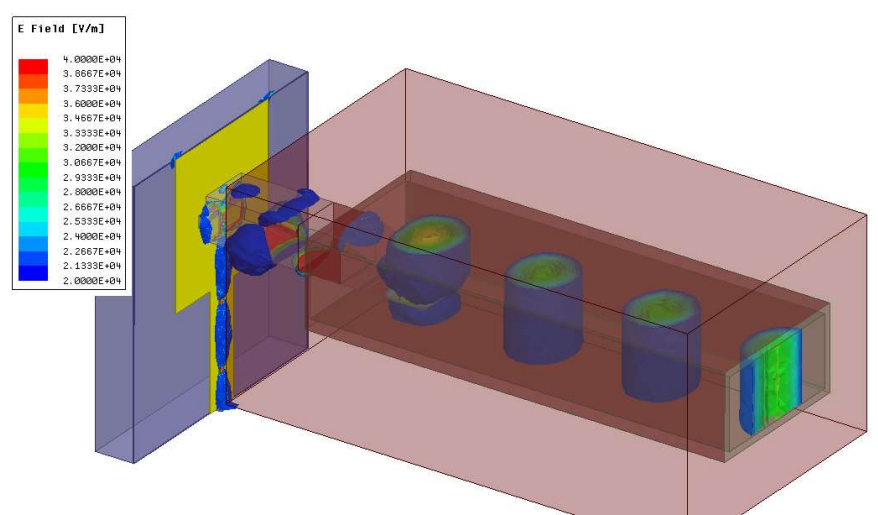

Fig. 3. EM simulation of the complete solution

epoxy. The holder dimensions and features are designed to provide accurate alignment of the taper to the air-filled waveguide.

HFSS 3D electromagnetic simulator was used to verify the performance of the structure as shown in Fig. 3. An air gap of 20 um between the antenna and the taper is included in simulations to model the non-galvanic contact effect. The Sparameters simulation showed an average insertion loss of 3.8 $\mathrm{dB}$. Simulations show that the main contributors to the loss are the eWLB mold dielectric loss with an average contribution of $0.95 \mathrm{~dB}$ and the radiation at the air gap between the antenna and the taper with a contribution of $0.71 \mathrm{~dB}$ to the total loss. Other sources of losses include the CPW feed lines and the dielectric loss of the taper and the adhesives used to assemble the interconnect. Simulation results are compared to measurements in section III.

\section{EXPERIMENTAL RESULTS AND DISCUSSION}

The fabricated slot antenna is shown in Figure 4. The feed lines for the antenna are implemented as CPW lines on the top exposed RDL allowing direct probing. The test setup consists of a Keysight PNA-X network analyzer and a pair of WR-6.5 VDI frequency extension modules to up/down-convert the

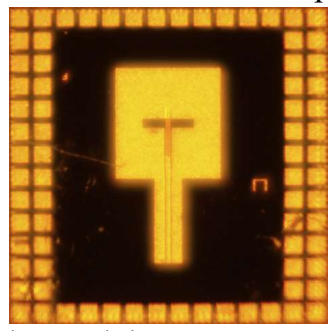

Fig. 4. Photo of the implemented slot antenna.

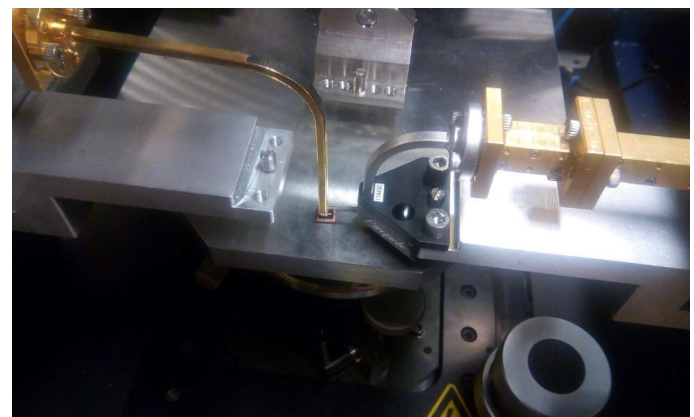

Fig. 5. Measurement setup for the interconnect with the waveguide bend on the left and waveguide probe on the right. 
signal to D-band. The measurement setup is shown in Figure 5. An open-ended waveguide bend is mounted perpendicular to the eWLB surface on one side of Cascade microtech's probe station allowing accurate positioning of the waveguide to the slot antenna. The taper along with the metal holder are attached to the open end of the waveguide bend. A D-band waveguide probe is mounted on the other side of the station to probe the CPW feed lines as mentioned earlier. Two-port calibration is performed to the outputs of the frequency extenders. The waveguide probe is de-embedded by measuring the S-parameters of the probe on a calibration substrate and de-embedding it using MATLAB. The waveguide bend is de-embedded by measuring a flanged waveguide of the same length and then de-emedding it in a similar fashion. De-embedded measurement results of the interconnect show an average insertion loss of $3.4 \mathrm{~dB}$ over the frequency range of $116-151 \mathrm{GHz}$ and a minimum of $2.1 \mathrm{~dB}$ as shown in Figure 6. The results show good agreement with simulations. Sensitivity to taper misalignment and the air gap to the eWLB surface was also simulated and compared to measurements. Results show that an air gap of up to $40 \mathrm{um}$ and a misalignment of $100 \mathrm{um}$ in both lateral directions can be tolerated. The interconnect shows a $3-\mathrm{dB}$ bandwidth of $26 \%$. The return losses at the WR-6.5 side and CPW lines side are shown in Figures 7 and 8 respectively.

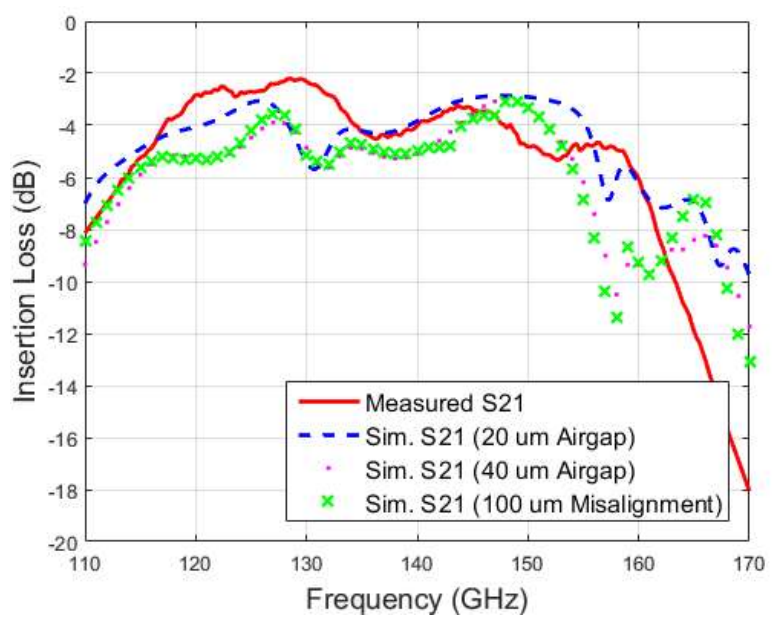

Fig. 6. The insertion loss of the interconnect

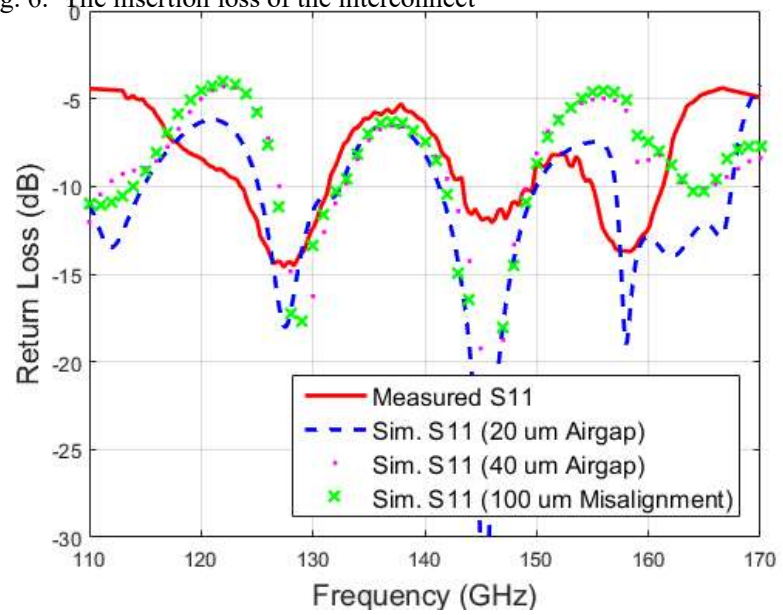

Fig. 7. The return loss of the interconnect at the WR-6.5 reference plane

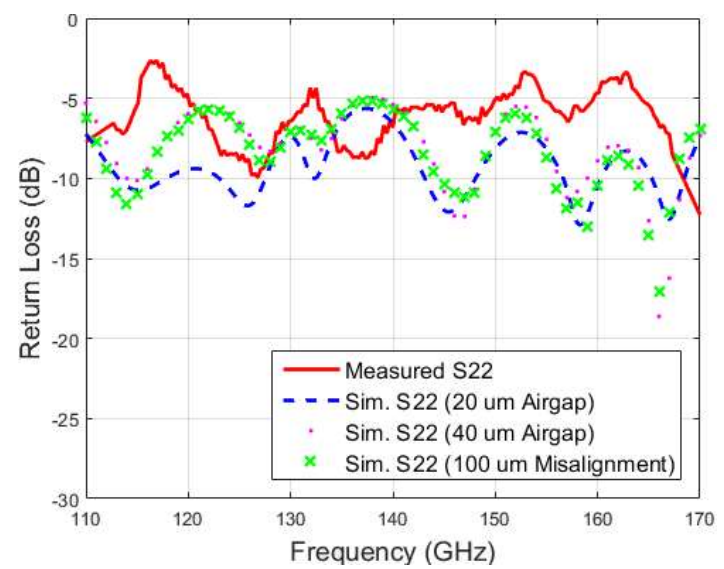

Fig. 8. The return loss of the interconnect at the $\mathrm{CPW}$ reference plane

\section{CONCLUSION}

A D-band MMIC-to-waveguide interconnect on eWLB process is presented. The interconnect consists of a slot antenna radiating to a HR Si taper inserted into an air-filled waveguide. The interconnect does not require galvanic contact between the antenna and the taper and represents a generic technique for integrating MMIC in $\mathrm{mmW}$ systems. Experimental results show that the interconnect achieves an average insertion loss of $3.4 \mathrm{~dB}$ and a bandwidth of $26 \%$ at Dband. The proposed interconnect addresses the integration challenge facing $\mathrm{mmW}$ systems and provides a low-cost solution that is implemented in a mass production commercial technology.

\section{ACKNOWLEDGMENT}

We would like to thank Ashraf Uz Zaman from Signals and Systems Dept. for technical discussions and Anna Malmros from Microwave Elec. Lab for her help to process the Si taper.

\section{REFERENCES}

[1] Peter H. Siegel, "Terahertz Technology," IEEE Trans. Microw. Theory Tech., vol. 50, no. 3, March 2002

[2] K. J. Lee, D. H. Lee, J. S. Rieh and M. Kim, "A V-Band Waveguide Transition Design Appropriate for Monolithic Integration," 2007 AsiaPacific Microwave Conference, Bangkok, 2007, pp. 1-4.

[3] W. R. Deal et al., "A $670 \mathrm{GHz}$ Low Noise Amplifier with $<10 \mathrm{~dB}$ Packaged Noise Figure," in IEEE Microwave and Wireless Components Letters, vol. 26, no. 10, pp. 837-839, Oct. 2016.

[4] V. Vassilev et al., "Integrated front-ends up to $200 \mathrm{GHz}$," 2011 IEEE MTT-S International Microwave Workshop Series on Millimeter Wave Integration Technologies, Sitges, 2011, pp. 57-60.

[5] L. Samoska et al., "Miniature packaging concept for LNAs in the 200$300 \mathrm{GHz}$ range," 2016 IEEE MTT-S International Microwave Symposium (IMS), San Francisco, CA, 2016, pp. 1-4.

[6] S. Beer et al., "Design and measurement of matched wire bond and flip chip interconnects for D-band system-in-package applications," 2011 IEEE MTT-S International Microwave Symposium, Baltimore, MD, 2011, pp. 1-4.

[7] M. Wojnowski et al., "Embedded Wafer Level Ball Grid Array (eWLB) Technology for Millimeter-Wave Applications", ECTC 2011

[8] E. Seler et al., "Chip-to-rectangular waveguide transition realized in embedded Wafer Level Ball Grid Array (eWLB) package", WAMICON 2014, Tampa, FL, 2014, pp. 1-4 